\title{
Synchronous gastric cancer and primary lymphoma of right adrenal gland: a case report
}

\author{
Shuichi Fukuda ${ }^{1}$ - Tomoko Wakasa $^{2} \cdot$ Hitoshi Hanamoto ${ }^{3} \cdot$ Taichi Koyama $^{1} \cdot$ Yoshio Ohta $^{2} \cdot$ Masatoshi Inoue $^{1}$. \\ Daisuke Terashita ${ }^{1}$ Atsushi Gakuhara ${ }^{1}$ Hideo Tomihara ${ }^{1} \cdot$ Katsuya Ohta $^{1} \cdot$ Kotaro Kitani $^{1} \cdot$ Kazuhiko Hashimoto $^{1}$. \\ Hajime Ishikawa ${ }^{1} \cdot$ Jin-ichi Hida ${ }^{1}$ Y Yutaka Kimura ${ }^{1}$
}

Received: 14 May 2021 / Accepted: 13 July 2021 / Published online: 20 July 2021

(c) The Author(s) 2021

\begin{abstract}
This report presents an extremely rare case of synchronous gastric cancer and primary adrenal diffuse large B-cell lymphoma (DLBCL). An 82-year-old man underwent computed tomography, which revealed a heterogeneous appearing and hypodense adrenal mass and a gastric mass with no enlarged lymph nodes in the neck, mediastinum, abdomen, and inguinal region. Upper gastrointestinal endoscopy revealed a protruding gastric tumor. The specimens obtained from endoscopic biopsy were histologically confirmed to be adenocarcinoma. The hormonal findings eliminated functional adrenal tumor. The patient underwent distal gastrectomy with regional lymph node resection for gastric cancer and incisional biopsy of the adrenal mass. Based on the pathological findings, diagnoses of mixed mucinous and tubular adenocarcinomas of the stomach and adrenal DLBCL were confirmed. Postoperation, the patient received rituximab combined with low-dose doxorubicin, cyclophosphamide, vincristine, and prednisone (R-miniCHOP). Six courses of R-miniCHOP were planned, but were completed in only one course at the patient's request. The patient died 2 months after surgery.
\end{abstract}

Keywords Adrenal lymphoma $\cdot$ Diffuse large B-cell lymphoma $\cdot$ Gastric cancer $\cdot$ Malignant lymphoma

\section{Introduction}

Gastric cancer is one of the most common gastrointestinal tract carcinomas. Alternatively, primary adrenal lymphoma is extremely rare, accounting for less than $1 \%$ of all nonHodgkin's lymphomas [1]. Primary adrenal lymphoma is based on a histologically confirmed lymphoma involving unilateral or bilateral adrenal glands without a previous history of lymphoma. Primary adrenal lymphoma requires a significant dominance of adrenal lesions when other organs or lymph nodes are involved by lymphoma concurrently with the adrenal glands [2].

Shuichi Fukuda

s.f4911@nifty.com

1 Department of Gastroenterological Surgery, Kindai University Nara Hospital, 1248-1, Otoda-cho, Ikoma, Nara 630-0293, Japan

2 Department of Pathology, Kindai University Nara Hospital, Nara, Japan

3 Department of Hematology, Kindai University Nara Hospital, Nara, Japan
Diffuse large B-cell lymphoma (DLBCL) is an aggressive lymphoma that accounts for about $30 \%$ of all cases of non-Hodgkin's lymphoma [3]. Among all cases of DLBCL, about $40 \%$ are extranodal [4]. The synchronous presence of gastric cancer and extranodal DLBCL is rare. In this study, we report an extremely rare case of synchronous gastric cancer and primary adrenal DLBCL.

\section{Case report}

An 82-year-old man was referred to our hospital for further investigation of a right adrenal mass and a gastric mass, which were coincidentally revealed by a follow-up computed tomography (CT) for an abdominal aortic aneurysm. The patient had a history of hypertension, diabetes mellitus, hyperlipidemia, cerebral infarction, and angina pectoris. The patient was an ex-smoker, consuming one pack per year for 55 years, and was a social drinker. In addition, the patient had a 6-kg weight loss in 1 month, abdominal pain, and anorexia. Physical examination showed no swelling of the superficial lymph nodes. The patient's blood pressure was 
$139 / 68 \mathrm{mmHg}$, pulse was 65 beats per minute, and body temperature was $36.8^{\circ} \mathrm{C}$. The patient had Eastern Cooperative Oncology Group performance status of 1 .

Noncontrast-enhanced CT revealed a heterogeneous appearing and hypodense adrenal mass with irregular margins and shape, $70 \mathrm{~mm}$ in size, and a gastric mass, $30 \mathrm{~mm}$ in size, with no enlarged lymph nodes in the neck, mediastinum, abdomen, and inguinal region (Fig. 1a, b). Contrast-enhanced CT revealed a slightly enhanced adrenal mass (Fig. 1c). Upper gastrointestinal endoscopy revealed a protruding gastric tumor in the gastric antrum of the posterior wall (Fig. 2). The specimens obtained from endoscopic biopsy were histologically confirmed to be adenocarcinoma. Laboratory data, including white blood cell count and sodium level, were within normal ranges. However, lactate dehydrogenase (LDH) level (274 U/L) was elevated in addition to microcytic hypochromic anemia (hemoglobin $9.5 \mathrm{~g} /$ dL). Tumor markers, including carcinoembryonic antigen, carbohydrate antigen 19-9, and $\alpha$-fetoprotein, were within normal ranges. The serum level of soluble interleukin-2 receptor (sIL-2R) was elevated $[916 \mathrm{U} / \mathrm{mL}$ vs. $<496 \mathrm{U} /$ $\mathrm{mL}$ (normal level)]. The plasma levels of adrenocorticotropic hormone, renin, adrenalin, dopamine, and cortisol were within the normal ranges. A decreased plasma level of aldosterone [18.3 pg/mL vs. $35.7-240.0 \mathrm{pg} / \mathrm{mL}$ (normal level)] and a decreased urinary level of vanillylmandelic acid [1.01 mg/day vs. $1.5-4.9 \mathrm{mg} /$ day (normal level)] were observed in addition to a slightly elevated plasma level of

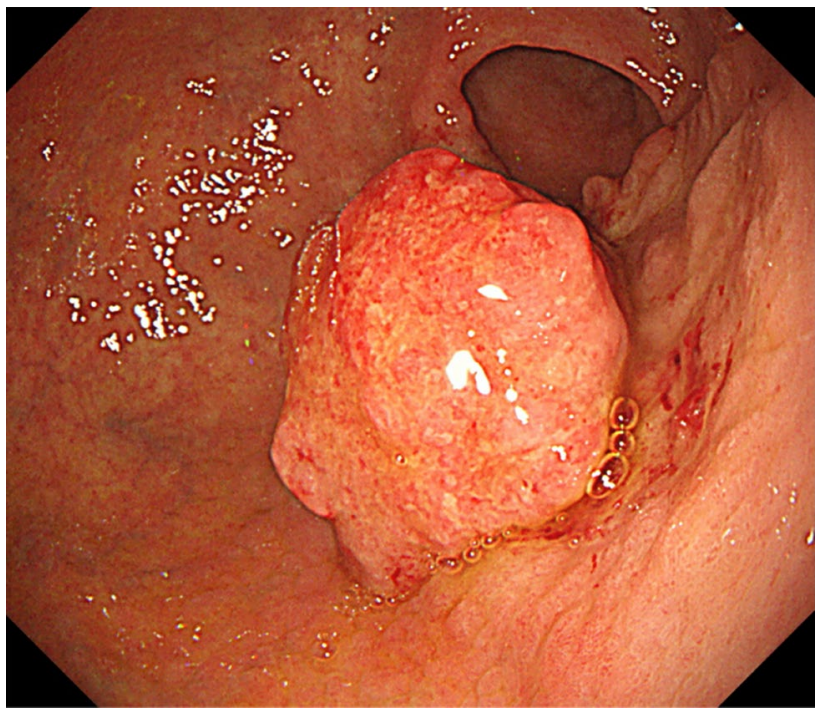

Fig. 2 Upper gastrointestinal endoscopy revealed a protruding gastric tumor in the gastric antrum of the posterior wall

noradrenaline $[0.59 \mathrm{ng} / \mathrm{mL}$ vs. $<0.5 \mathrm{ng} / \mathrm{mL}$ (normal level)]. These hormonal findings eliminated the diagnosis of functional adrenal tumor. The adrenal mass was large and nonfunctional, it revealed irregular margins and shape, and enhancement on CT and was coexistent with gastric cancer, and the sIL-2R level was elevated; therefore, we considered malignant tumors, including adrenal metastasis from gastric
Fig. 1 a, b Noncontrastenhanced CT revealed a heterogeneous appearing and hypodense adrenal mass with irregular margins and shape (a, arrow), $70 \mathrm{~mm}$ in size, and a gastric mass (b, arrow), $30 \mathrm{~mm}$ in size, with no enlarged lymph nodes. c Contrast-enhanced CT revealed a slightly enhanced adrenal mass
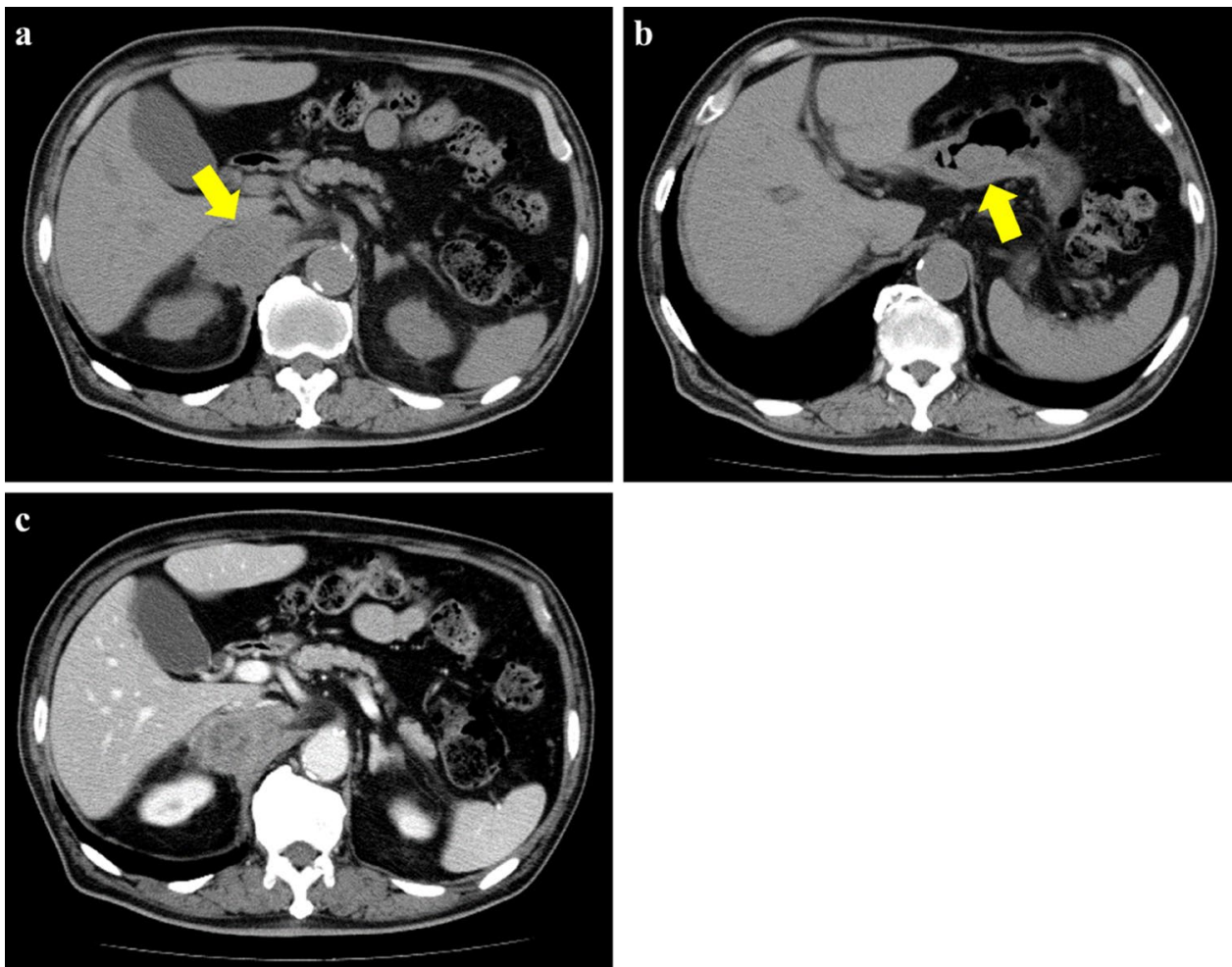
cancer, adrenocortical carcinoma, and adrenal lymphoma. After a discussion at a multidisciplinary cancer conference, gastrectomy, and right adrenalectomy were planned.

Surgical exploration of the abdomen revealed no evidence of ascites or metastasis to the liver or peritoneum. Distal gastrectomy with regional lymph node resection was performed for gastric cancer. The adrenal gland mass was tightly adherent to the surrounding tissues; therefore, the mass was judged to be impossible to resect, and an incisional biopsy was performed.

Hematoxylin and eosin staining of the adrenal mass showed a diffuse proliferation of large and atypical lymphocytes accompanied by small lymphocytes (Fig. 3a). Immunohistochemical staining revealed that large and atypical lymphocytes were positive for CD20 (Fig. 3b), bcl-2, and leukocyte common antigen and negative for CD3 (Fig. 3c), cytokeratin AE1/AE3 (Fig. 3d), chromogranin A, synaptophysin, desmin (Fig. 3e), myeloperoxidase, CD34, and IgG4. These histopathological and immunohistochemical findings were consistent with DLBCL. The size of the resected gastric specimen was $50 \times 50 \mathrm{~mm}$ (Fig. 4a). Hematoxylin and eosin staining of the gastric mass revealed mixed mucinous and tubular adenocarcinomas (Fig. 4b). HER2 immunohistochemical staining was negative. No Helicobacter pylori were detected by Giemsa staining in the resected specimens. The resection margins were free of tumor cells. The tumor had invaded the submucosal layer at a depth of $4 \mathrm{~mm}$ in the submucosa. The resected regional lymph nodes revealed no evidence of metastasis. The patient was diagnosed with T1bN0M0, stage IA according to the 8th edition of the Union for International Cancer Control TNM Classification of Malignant Tumors.

Bone marrow aspiration indicated no bone marrow invasion of DLBCL. The patient had stage IE according to the Ann Arbor staging system. According to the National Comprehensive Cancer Network International Prognostic Index [5], two adverse factors were applicable [age of more than 75 years ( 3 points) and LDH ratio of more than 1-3 (1 point)], and the risk group was high-intermediate (4 points). The patient received rituximab combined with low-dose doxorubicin, cyclophosphamide, vincristine, and prednisone (R-miniCHOP). The size of the adrenal tumor
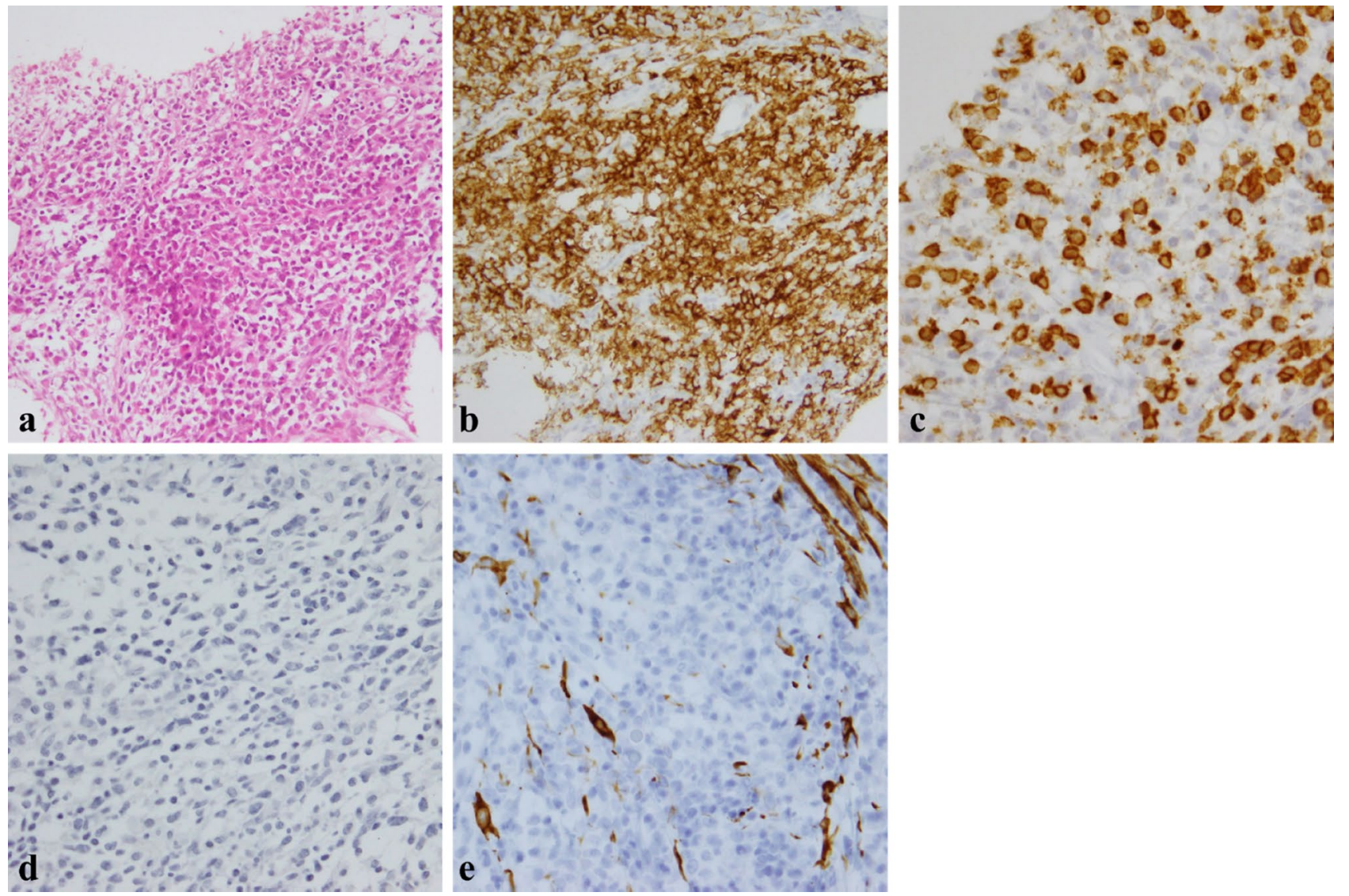

Fig. 3 a Hematoxylin and eosin staining of the adrenal mass showed a diffuse proliferation of large and atypical lymphocytes accompanied with small lymphocytes. b-e Immunohistochemical staining revealed that large and atypical lymphocytes were positive for CD20 (b) and negative for CD3 (c), cytokeratin AE1/AE3 (d), and desmin (e). The small CD3 + non-lymphoma T cells form the cellular infiltrate around the large and atypical lymphoma B cells (c) 
Fig. 4 a The size of the resected gastric specimen was $50 \times 50 \mathrm{~mm}$. b Hematoxylin and eosin staining of the gastric mass showed mixed mucinous and tubular adenocarcinomas
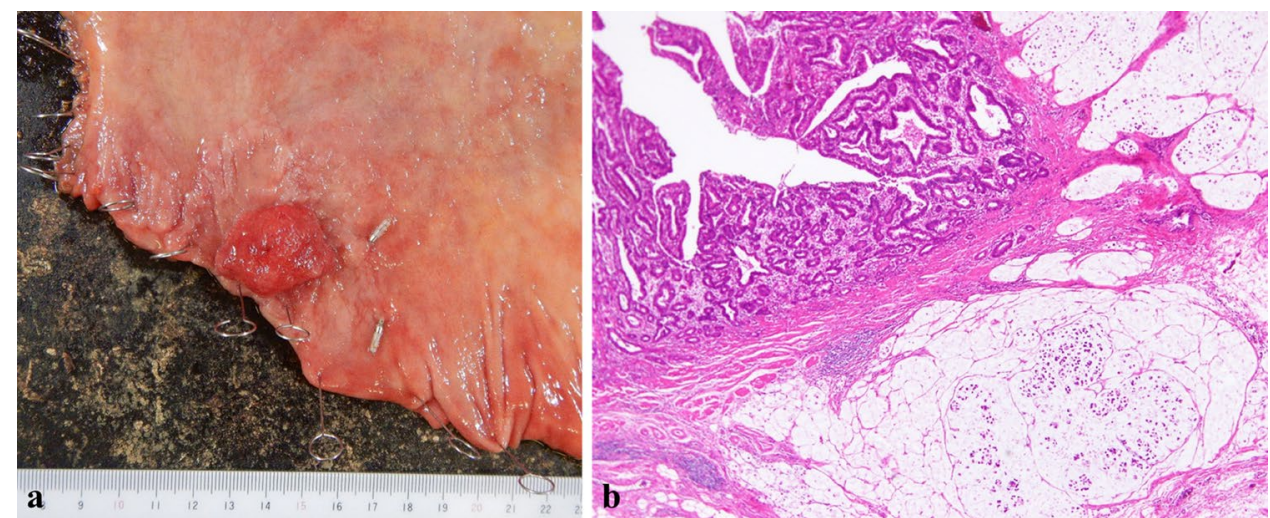

was unchanged on CT after one course of R-miniCHOP. Six courses of R-miniCHOP were planned but were completed in only one course at the patient's request. Then, the patient was transferred to a palliative care ward of a hospital and palliative medicine was introduced. The patient died 2 months after surgery.

\section{Discussion}

Gastric cancer is the fourth most common cancer and the second most common cause of cancer-related death worldwide [6]. Alternatively, about 150,000 new cases of DLBCL annually occur worldwide, representing about $30 \%$ of all cases of non-Hodgkin's lymphoma [3]. DLBCLs can occur in any organ, and about $40 \%$ of DLBCL cases are extranodal [4]. Synchronous multiple primary cancers are defined as two or more tumors occurring within 6 months of each other [7], and among them, synchronous gastric cancer and primary extranodal DLBCL are rare. To the best of our knowledge, 13 cases of synchronous gastric cancer and primary gastric DLBCL and two cases of synchronous gastric cancer and primary small intestinal DLBCL have been reported in English [8-23]. Particularly, the patient in this case report is the first reported case of synchronous gastric cancer and primary adrenal DLBCL in the English literature.

With the development of diagnostic imaging, the number of adrenal tumors found by chance is increasing [24, 25]. Among adrenal lesions larger than $5 \mathrm{~cm}$ in diameter on CT, $28.69 \%$ are malignant adrenal tumors, including adrenal lymphoma, accounting for $1.20 \%$ of all cases [26]; however, CT findings of primary adrenal lymphoma are not specific. Rashidi et al. have reported that in CT studies of primary adrenal lymphomas, the frequency of homogeneous and heterogeneous appearing masses is similar; most masses are hypodense, whereas some are hyperdense; and most enhancement patterns are "slight to moderate enhancement," whereas some are "no or poor enhancement [27]." Therefore, a definitive diagnosis is only established by resection or biopsy [27]. In the patient in this case report, CT revealed a "heterogeneous appearing", "hypodense", and "slightly enhanced" adrenal mass with a size of $70 \mathrm{~mm}$. Although these CT findings are relatively common in adrenal primary lymphoma, no definitive diagnosis could be made based on imaging studies alone, and an intraoperative biopsy provided a definitive diagnosis. On magnetic resonance imaging (MRI), primary adrenal lymphomas generally show a hypointense pattern on T1-weighted imaging and a hyperintense pattern on T2-weighted imaging [27]. Furthermore, positron emission tomography (PET) is useful in monitoring treatment response and detecting recurrence [27]. Regretfully, both MRI and PET were not performed on the patient in this case report.

The adrenal gland is an organ with abundant blood flow and is prone to hematogenous metastasis from malignant tumors [28]. Adrenal metastasis is not rare in advanced gastric cancer, as there are also reports of adrenal metastasis in early gastric cancer $[29,30]$. Therefore, although the preoperative size of gastric cancer was not large in this study, adrenal metastasis from gastric cancer was considered a differential diagnosis of adrenal tumors on imaging. Similar to adrenal lymphoma, metastatic adrenal tumors do not have characteristic CT findings [24, 25]. Gastric cancers with distant metastasis are usually non-candidate for surgery, and chemotherapy is the standard of care for those cases if no tumor bleeding or gastrointestinal obstruction exists; however, for gastric cancers with simultaneous adrenal metastasis, gastrectomy, and adrenalectomy have been reported to provide long-term survival [30]. Therefore, we planned to perform gastrectomy and adrenalectomy for gastric cancer without tumor bleeding or gastrointestinal obstruction combined with a suspicious adrenal metastasis from gastric cancer. The adrenal mass was finally confirmed to be adrenal lymphoma according to pathological findings. This case indicates that when an adrenal mass is simultaneously found with gastric cancer, the adrenal mass should be resected or biopsied to make a definitive diagnosis without assuming that the mass is an adrenal metastasis from gastric cancer. 
In the present study, incisional biopsy of the adrenal mass was performed concurrently with gastrectomy for gastric cancer. The adrenal mass was diagnosed as a primary adrenal lymphoma, a disease with poor prognosis, and gastric cancer was pathologically diagnosed as stage IA. Through gastrectomy, chemotherapy for primary adrenal lymphoma had become less tolerable. The preoperative gastric cancer was not large in size and had no enlarged lymph nodes, and there was no tumor bleeding or gastrointestinal obstruction. The gastric cancer was of much less concern than the adrenal mass on imaging. Therefore, in the present case, the histopathological confirmation of the adrenal mass should have been performed first by diagnostic laparoscopy without gastrectomy. In this way, the diagnosis of the adrenal mass could have been made less invasive, and chemotherapy would not have been less tolerable by preserving the stomach. When gastric cancer and an adrenal mass are found simultaneously, the diagnostic approach of the adrenal mass needs to be carefully considered, taking into account the malignancy and progression of each tumor, and when gastric cancer is of much less concern than the adrenal mass as in this case, it is necessary to make a diagnosis of the adrenal mass first before treating the gastric cancer.

Secondary involvement of the adrenal glands by a malignant lymphoma has been reported to occur in as many as $25 \%$ of patients with the disease [31]. However, primary adrenal lymphoma is rare, accounting for $3 \%$ of all cases of primary extranodal lymphomas [1]. Among primary adrenal lymphomas, the most frequent histology is DLBCL, accounting for 76.3-78.6\% of all cases, and common symptoms include B symptoms (fever, night sweats, and weight loss), fatigue, abdominal pain, and anorexia, which are consistent with the patient in this case report [27, 32, 33]. Primary adrenal DLBCL is a disease of the elderly, with a median age at diagnosis of 71 years, and more likely to affect men, with a male-to-female ratio of $2: 1$, which are also consistent with the patient in this case report [1]. The median survival of patients with primary adrenal DLBCL is poor, being only 14 months [1]. In this study, the primary adrenal lymphoma was right unilateral, and the patient did not show adrenal insufficiency, including hyponatremia and hypotension. This finding agreed with the previous studies that all patients with confirmed adrenal insufficiency had bilateral adrenal lymphomas, and no patients with unilateral adrenal lymphoma showed adrenal insufficiency $[32,34]$. Chemotherapy with R-CHOP is the widely recognized treatment strategy for primary adrenal lymphoma [2]. R-miniCHOP was administered to the patient in this case report because the patient was elderly, had just undergone gastrectomy, and had multiple comorbidities [35]. Adrenal involvement is a known risk factor for central nervous system relapse [36, 37]. In this study, the patient was transferred to a different hospital, so whether central nervous system relapse occurred or not is unclear.

Table 1 shows 16 cases of synchronous gastric cancer and extranodal DLBCL previously reported in English, including the patient in this case report $[8-19,22,23]$. Fourteen of the 16 patients $(87.5 \%)$ were male, with a median age of 71 years (range, $47-82$ years). The tumor depth of gastric cancer among the 14 lesions (13 patients) was as follows: mucosa, seven lesions (50.0\%); submucosa, two lesions (14.3\%); muscularis propria, three lesions (21.4\%); tumor penetration of the serosa, one lesion (7.1\%); and tumor invasion of adjacent structures, one lesion (7.1\%). The median tumor size of DLBCL was $65 \mathrm{~mm}$ (range, 25-130 mm). In Table 1, one patient (case 7) was in poor condition and could not be treated, one patient (case 8) was not a candidate for chemotherapy because of comorbidities, one patient (case 12) underwent only chemotherapy because diagnostic laparoscopy showed peritoneal carcinomatosis of gastric cancer, one patient (case 13) rejected chemotherapy for DLBCL, and we did not know whether cases 2 and 5 underwent chemotherapy because it was not mentioned in the paper. The other 10 of the 16 patients $(62.5 \%)$ underwent tumor resection (operation or endoscopic resection) and chemotherapy. As in case 1, long-term survival of more than 120 months is expected with appropriate treatment.

As this study was a single-patient case report, the accumulation of prospective evidence from more patients in multiple institutions should confirm the findings of this case report. Primary adrenal DLBCL are relatively rare; therefore, the number of patients treated in a single institution is limited. However, the current findings provide important information that can contribute to the development of a treatment strategy for the concomitant occurrence of gastric cancer and adrenal mass.

In conclusion, this case report detailed the case of a patient with synchronous gastric cancer and primary lymphoma of right adrenal gland. This case indicates that in patients with concomitant gastric cancer and an adrenal mass, clinicians should be careful not to assume that the adrenal mass is an adrenal metastasis from gastric cancer without histopathological confirmation and need to consider the possibility of adrenal lymphoma. Furthermore, clinicians need to carefully consider the diagnostic approach of the adrenal mass, taking into consideration the malignancy and progression of each tumor. 


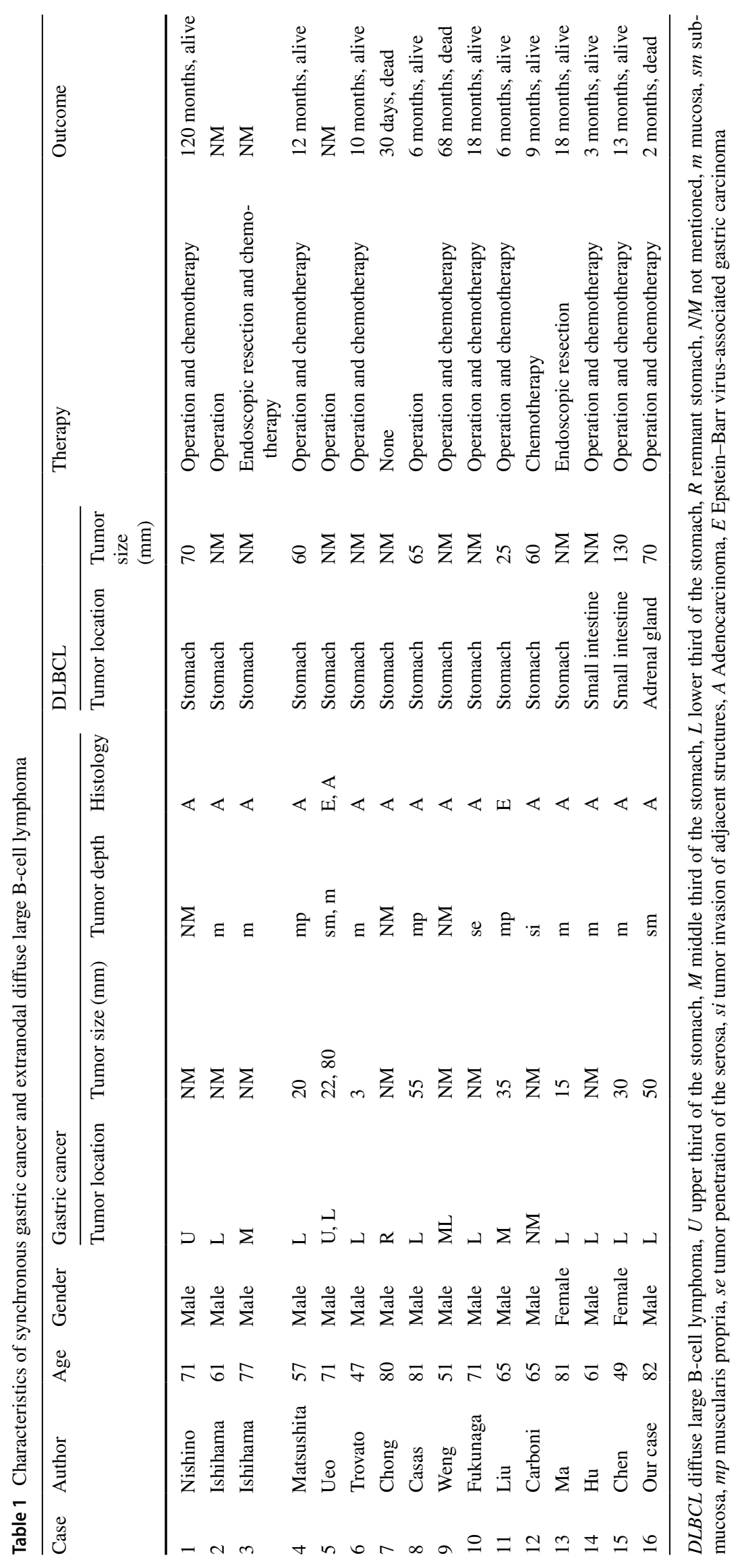


Acknowledgements The authors would like to thank Enago (www. enago.jp) for the English language review.

Author contributions SF conceived and designed the study. SF, TW, $\mathrm{HH}$, and YO acquired the data. SF, TK, MI, DT, AG, HT, KO, KK, $\mathrm{KH}, \mathrm{HI}, \mathrm{JIH}$, and $\mathrm{YK}$ analyzed and interpreted the data. SF drafted the manuscript. SF, TW, HH, and YO revised the manuscript critically for important intellectual content. SF, TW, HH, and YO are responsible for confirming the authenticity of all the raw data. All authors read and approved the final manuscript.

\section{Declarations}

Conflict of interest Shuichi Fukuda, Tomoko Wakasa, Hitoshi Hanamoto, Taichi Koyama, Yoshio Ohta, Masatoshi Inoue, Daisuke Terashita, Atsushi Gakuhara, Hideo Tomihara, Katsuya Ohta, Kotaro Kitani, Kazuhiko Hashimoto, Hajime Ishikawa, Jin-ichi Hida and Yutaka Kimura declare that they have no conflict of interest.

Ethical approval This study was approved by the Ethics Committee of our institution (approval number: 21-04).

Human rights All procedures followed have been performed in accordance with the ethical standards laid down in the 1964 Declaration of Helsinki and its later amendments.

Informed consent Informed consent was obtained from all patients for being included in the study.

Open Access This article is licensed under a Creative Commons Attribution 4.0 International License, which permits use, sharing, adaptation, distribution and reproduction in any medium or format, as long as you give appropriate credit to the original author(s) and the source, provide a link to the Creative Commons licence, and indicate if changes were made. The images or other third party material in this article are included in the article's Creative Commons licence, unless indicated otherwise in a credit line to the material. If material is not included in the article's Creative Commons licence and your intended use is not permitted by statutory regulation or exceeds the permitted use, you will need to obtain permission directly from the copyright holder. To view a copy of this licence, visit http://creativecommons.org/licenses/by/4.0/.

\section{References}

1. Li S, Wang Z, Wu Z, et al. Clinical characteristics and outcomes of primary adrenal diffuse large B cell lymphoma in a large contemporary cohort: a SEER-based analysis. Ann Hematol. 2019;98:2111-9.

2. Yang Y, Xie W, Ren Y, et al. A case report of primary adrenal lymphoma: a rare but aggressive and invasive disease. Medicine (Baltimore). 2020;99: e20938.

3. Sehn LH, Salles G. Diffuse large B-cell lymphoma. N Engl J Med. 2021;384:842-58.

4. Møller MB, Pedersen NT, Christensen BE. Diffuse large B-cell lymphoma: clinical implications of extranodal versus nodal presentation - a population-based study of 1575 cases. Br J Haematol. 2004;124:151-9.

5. Zhou Z, Sehn LH, Rademaker AW, et al. An enhanced International Prognostic Index (NCCN-IPI) for patients with diffuse large B-cell lymphoma treated in the rituximab era. Blood. 2014;123:837-42.
6. Bang YJ, Van Cutsem E, Feyereislova A, et al. Trastuzumab in combination with chemotherapy versus chemotherapy alone for treatment of HER2-positive advanced gastric or gastro-oesophageal junction cancer (ToGA): a phase 3, open-label, randomised controlled trial. Lancet. 2010;376:687-97.

7. Meng J, Pan H, Li X, et al. Diagnosis and treatment of synchronous lymphoma and digestive system carcinoma: report of four cases and literature review. Front Oncol. 2019;9:1367.

8. Nishino N, Konno H, Baba S, et al. Synchronous lymphoma and adenocarcinoma occurring as a collision tumor in the stomach: report of a case. Surg Today. 1996;26:508-12.

9. Ishihama T, Kondo H, Saito D, et al. Clinicopathological studies on coexisting gastric malignant lymphoma and gastric adenocarcinoma: report of four cases and review of the Japanese literature. Jpn J Clin Oncol. 1997;27:101-6.

10. Matsushita M, Hajiro K, Okazaki K, et al. Simultaneous adenocarcinoma and malignant lymphoma of the stomach. Endoscopy. 1997;29:134.

11. Ueo T, Kashima K, Daa T, et al. Coexistence of Epstein-Barr virus-associated gastric carcinoma with malignant lymphoma: report of two cases. Virchows Arch. 2006;449:215-9.

12. Trovato C, Sonzogni A, Ravizza D, et al. Confocal laser endomicroscopy diagnosis of gastric adenocarcinoma in a patient treated for gastric diffuse large-B-cell lymphoma. Dig Liver Dis. 2009;41:447-9.

13. Chong VH, Idros A, Telisinghe PU. Triple synchronous gastrointestinal malignancies: a rare occurrence. Singapore Med J. 2010;51:e176-8.

14. Casas CO, Guillén VV, Tarragó AC, et al. Synchronic gastric adenocarcinoma and lymphoma. Rev Esp Enferm Dig. 2011;103:388-9.

15. Weng CH, Wang RC, Wu CC, et al. Treatment of synchronous adenocarcinoma and lymphoma of the stomach: a case report. Mol Clin Oncol. 2016;5:783-5.

16. Fukunaga $\mathrm{H}$, Asama $\mathrm{H}$, Kaneda $\mathrm{H}$, et al. Collision tumor with diffuse large B cell lymphoma and gastric cancer. Jpn J Clin Oncol. 2016;46:968-9.

17. Liu L, Zhao H, Sheng L, et al. Collision of lymphoepitheliomalike carcinoma with diffuse large B-cell lymphoma of the stomach: a case report. Anticancer Res. 2017;37:4569-73.

18. Carboni F, Diodoro MG, Valle M. Synchronous primary diffuse large B cell lymphoma and adenocarcinoma of the stomach: a clinical dilemma. Indian J Surg Oncol. 2020;11:S223-5.

19. Ma YH, Yamaguchi T, Yasumura T, et al. Pancreatic cancer secondary to intraductal papillary mucinous neoplasm with collision between gastric cancer and B-cell lymphoma: a case report. World J Clin Cases. 2021;9:2400-8.

20. Hamaloglu E, Topaloglu S, Ozdemir A, et al. Synchronous and metachronous occurrence of gastric adenocarcinoma and gastric lymphoma: a review of the literature. World J Gastroenterol. 2006;12:3564-74.

21. Namikawa T, Munekage E, Fukudome I, et al. Clinicopathological characteristics and therapeutic outcomes of synchronous gastric adenocarcinoma and gastric lymphoma. Anticancer Res. 2014;34:5067-74.

22. $\mathrm{Hu} \mathrm{XR}, \mathrm{Hu} \mathrm{YX}, \mathrm{Fu} \mathrm{HR}$, et al. Diffuse large B-cell lymphoma with concurrent gastric adenocarcinoma: case report and literature review. J Int Med Res. 2011;39:2051-8.

23. Chen DW, Pan Y, Yan JF, et al. Laparoscopic resection of synchronous gastric cancer and primary small intestinal lymphoma: a case report. World J Gastroenterol. 2014;20:6353-6.

24. Young WF Jr. Clinical practice. The incidentally discovered adrenal mass. N Engl J Med. 2007;356:601-10.

25. Kebebew E. Adrenal incidentaloma. N Engl J Med. 2021:384:1542-51. 
26. Zhang Z, Wang L, Chen J, et al. Clinical analysis of adrenal lesions larger than $5 \mathrm{~cm}$ in diameter (an analysis of 251 cases). World J Surg Oncol. 2019;17:220.

27. Rashidi A, Fisher SI. Primary adrenal lymphoma: a systemic review. Ann Hematol. 2013;92:1583-93.

28. Zerrweck C, Caiazzo R, Clerquin B, et al. Renal origin and size are independent predictors of survival after surgery for adrenal metastasis. Ann Surg Oncol. 2012;19:3621-6.

29. Igami T, Yamaguchi A, Isogai M, et al. A resected case of early gastric cancer with synchronous adrenal metastasis. Jpn J Cancer Clin. 1998;44:833-7 (In Japanese).

30. Kosaka T, Ueshige N, Sugaya JI, et al. A long surviving case of resected gastric cancer presenting with a synchronous adrenal metastasis. Gastric Cancer. 1999;2:142-4.

31. Wang J, Sun NC, Renslo R, et al. Clinically silent primary adrenal lymphoma: a case report and review of the literature. Am J Hematol. 1998;58:130-6.

32. Laurent C, Casasnovas O, Martin L, et al. Adrenal lymphoma: presentation, management and prognosis. QJM. 2017;110:103-9.

33. Majidi F, Martino S, Kondakci M, et al. Clinical spectrum of primary adrenal lymphoma: results of a multicenter cohort study. Eur J Endocrinol. 2020;183:453-62.
34. McDonnell T, Mulkerrin E. Primary adrenal lymphoma. N Engl J Med. 2021;384:165.

35. Peyrade F, Jardin F, Thieblemont C, et al. Attenuated immunochemotherapy regimen (R-miniCHOP) in elderly patients older than 80 years with diffuse large B-cell lymphoma: a multicentre, single-arm, phase 2 trial. Lancet Oncol. 2011;12:460-8.

36. Schmitz N, Zeynalova S, Nickelsen M, et al. CNS International Prognostic Index: a risk model for CNS relapse in patients with diffuse large B-cell lymphoma treated with R-CHOP. J Clin Oncol. 2016;34:3150-6.

37. Harrysson S, Eloranta S, Ekberg S, et al. Incidence of relapsed/ refractory diffuse large B-cell lymphoma (DLBCL) including CNS relapse in a population-based cohort of 4243 patients in Sweden. Blood Cancer J. 2021;11:9.

Publisher's Note Springer Nature remains neutral with regard to jurisdictional claims in published maps and institutional affiliations. 